\title{
A Modified APACHE II Score for Predicting Mortality of Variceal Bleeding
}

El Nibras M Abdelrahim*, Adam AM*, Ibnouf MAM ${ }^{*}$

\section{Abstract}

Introduction: Prediction of outcome of based on personal experience is subjective. There is a need for a method of prediction to suit our current state of practice.

Objectives: To evaluate a modified APACHE II system in predicting mortality in acute variceal bleeding.

Patients and methods: Prospective study conducted from June 2005 through September 2006 in the Ibn Sina Hospital Bleeding Centre ICU. Clinical and laboratory data for 102 patients were allotted a modified APACHE II score, a child-Pugh class and fed to SPSS to calculate predicted mortality.

Results: The mean $( \pm$ SD) age $49.2( \pm 16.1)$ years. Male were $79(77.5 \%)$. Patients with liver cirrhosis and periportal fibrosis were $75(73.5 \%)$ and $27(26.5 \%)$ ) respectively. The mean predicted mortality was $22.8 \%$ while the actual mortality was $26.5 \%(27)$ patients. The mean modified APACHE II score among the deceased group was 19.04 . Rate of deaths among liver cirrhosis patients was $12(44.4 \%)$ with mean predicted mortality of $27.3 \%$. Deaths among periportal fibrosis occur at mean modified APACHE II score 17.7 and mean predicted mortality of $20 \%$. Seventeen patients had ICU stay $\geq 4$ days. Ten out of them died $(58.8 \%)$.

Conclusion: Modified APACHE II score is effective in predicting outcome of patients with variceal bleeding. Score of $\geq 15$ points and long ICU stay are associated with high mortality.

Keywords: liver cirrhosis, periportal fibrosis, portal hypertension, schistosomiasis,

\section{Introduction}

Predicting outcome of patients with massive variceal bleeding is subjective if it is not based on specific outcome measures. Late in the $20^{\text {th }}$ century, a variety of objective scoring systems have been developed in an attempt to estimate prognosis more accurately using criteria derived from large patients data base. It has been clearly demonstrated that during critical illness the degree of derangement of physiological variables is closely related to subsequent hospital mortality.

In mid 1970s William Knaus ${ }^{1}$ developed the APACHE (Acute physiology, Age and Chronic Health Evaluation) scoring system. Initially 34 physiological variables, which were thought to have effect on outcome were selected (APACHE I). Later on it had been reduced to 12 more commonly measured variables (APACHE II), which was introduced in $1985^{2,3}$. The total number of points gives a score ranging from 0 71.The higher the score the greater the severity of illness.

Because of lack of facilities we used in this prospective study a modified APACHE II score to test its value in predicting mortality in patients suffering of severe acute variceal bleeding who were admitted to the ICU at the Ibn Sina Hospital. The modifications made to the standard APACHE II score were:

1- Measurement of blood PH was omitted because of lack of blood gas analysis machine at the time of the study.

*E1 Nibras M AbdelRahim\& Ahmed Adam Mohamed, MAM Ibnouf Surgeons at Ibn Sina Hospital
2- To calculate inspired oxygen; the oxygen saturation was noted from oximeter and converted through oxygen dissociation curve.

\section{Objectives}

To determine the effectiveness of a modified APACHE II score in predicting the outcome of patients, admitted to ICU of the GIT Bleeding Centre at Ibn Sina Hospital in Khartoum, suffering of acute oesophageal variceal bleeding and to find out high risk factors associated with such mortality.

\section{Methods}

This is a prospective study done between June 2005 and Sept. 2006. All patients with massive oesophageal variceal bleeding were considered for the study. Informed consents were obtained and ethical clearance was granted. Patients with upper GIT bleeding due to causes other than variceal bleeding and patients who do not meet the eligibility criteria of modified APACHE II score and Child-Pugh classification within the first 24 hours of admission were excluded. The studied population consists of 102 critically ill patients with oesophageal variceal bleeding.

Set up: This study was conducted at the ICU of GIT bleeding centre at Ibn Sina Hospital. The GIT Bleeding Centre consists of well equipped ICU, intermediate care unit with 4 beds, general ward, full equipped endoscopic theatre, blood bank, laboratory facilities, pharmacy, upper and lower GIT emergency endoscopic service and elective GIT manometry. The ICU staffs consist of 3 to 4 
sisters, one registrar, one medical officer, and one consultant per duty. It receives critically ill patients with upper and lower GIT bleeding who were referred from other hospitals.

Clinical and laboratory data conforming to the modified APACHE II score and Child-Pugh classification were collected in the first 24 hours of admission. This include age, sex, medical history, mean BP, pulse rate, temperature, RR, GCS, presence of ascites, jaundice, serum creatinine, total bilirubin, serum electrolytes, total $\mathrm{WBC}, \mathrm{Hb}$, oxygen saturation, prothrombin time and serum albumin.

\section{Statistical analysis}

Data were fed to Statistical Package of Social Sciences (SPSS) program for descriptive and analytic statistics. The level of significance was taken at $P<0.05$.

\section{Results}

102 patients were studied. They were 79 $(77.5 \%)$ males and $23(22.5 \%)$ females with mean $( \pm$ SD) age $49.2( \pm 16.1)$ range $(18-89)$ years. The cause of portal hypertension in this studied group was liver cirrhosis in $27(26.5 \%)$ patients, and periportal fibrosis in $75(73.5 \%)$ patients. Their modified APACHE II score ranged from 5 to 29 with mean $( \pm$ SD) $14.96( \pm 5.3)$. Their predicted mortality ranged from $8 \%$ to $40 \%$ with mean of $22.8 \%$. The actual mortality was (27) $26.5 \%$ patients. Among the deaths $15(14.7 \%)$ patients had periportal fibrosis and $12(11.8 \%)$ patients had cirrhosis. The modified APACHE II score among the deceased group ranged from 7 to 29 with a mean of 19. In contrast the modified APACHE II for the survival group, ranged from 5 to 23 with a mean of 13.6. However, patients who had modified APACHE II score $\geq 15$ were $54(52 \%)$ patients but 23 patients out of them died.

Child-Pugh classification was also used as disease specific scoring system so as to find out its prediction of mortality. Child class A patients were $43(42.2 \%)$ with only three deaths, which represented $2.9 \%$ of the studied population. Patients with Child class B were $38(37.3 \%)$ with 10 deaths, which represented $9.8 \%$ of the total mortality. Those with Child class $\mathrm{C}$ were $21(20.8 \%)$ patients with 14 deaths, which represented $13.7 \%$ of the total mortality (figure 1 ).

There were 27 patients with liver cirrhosis $12(44.4 \%)$ out of them died. Their modified APACHE II score ranged from 11 to 29 with a mean of 20 and mean predicted mortality rate $27.3 \%$. Nine out of the 12 patients who died in the
ICU were Child-Pugh classification class $C$. The rest of the deaths of cirrhosis were two with Child $B$ class and only one patient with Child A class.

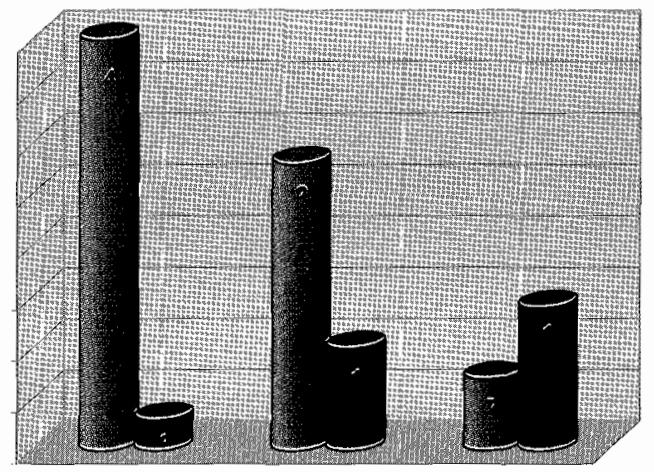

Fig: Relation between Child-Pugh classification and death

There were 75 patients with periportal fibrosis, 15 out of them died giving a mortality rate of $20 \%$. Patients with periportal fibrosis showed modified APACHE II score predicted mortality rate of 17.7. Child-Pugh classification of deaths in this group were Child class B in eight $(53.3 \%)$ patient, Child class $\mathrm{C}$ in five $(33 \%)$ patients, and Child class $\mathrm{A}$ in two(13.3\%) patients.

Relationship between co-morbid diseases and deaths:

\section{Patients with liver cirrhosis:}

In 27 patients with liver cirrhosis, 21 $(77.8 \%)$ patients had no associated disease. Nine out of those patients $(42.9 \%)$ died. The remaining six patients $(22.2 \%)$ with liver cirrhosis had associated co-morbid disease; three patients out of them $(50 \%)$ died (Table 1$)$.

Table 1. Relation of associated disease to mortality in patients with liver cirrhosis

\begin{tabular}{|c|c|c|c|c|}
\hline Total & \multicolumn{2}{|c|}{$\begin{array}{l}\text { Liver cirrhosis } \\
\text { without co-morbid } \\
\text { disease }\end{array}$} & \multicolumn{2}{|c|}{$\begin{array}{l}\text { Liver cirrhosis } \\
\text { with co-morbid } \\
\text { disease }\end{array}$} \\
\hline \multirow[t]{2}{*}{27} & $21(77.8 \%)$ & & $6(22.2 \%$ & \\
\hline & $\begin{array}{l}\text { Alive } \\
12(57.1 \%)\end{array}$ & $\begin{array}{l}\text { Died } \\
9(42.9 \%)\end{array}$ & $\begin{array}{l}\text { Alive } \\
3(50 \%)\end{array}$ & Died \\
\hline
\end{tabular}

\section{Patients with periportal fibrosis:}

Total number of patients with periportal fibrosis was 75 patients. $55(73.3 \%)$ of them had no associated disease. Eight patients from this group died and 47 patients remained alive. The number of patients with periportal fibrosis and associated co-morbid disease were $20(26.7 \%)$, seven out of them died and 13 patient remained alive (Table 2). 
Table (2) Relation of associated disease to mortality in patients with periportal fibrosis

\begin{tabular}{|c|c|c|c|c|}
\hline Total & $\begin{array}{l}\text { Periportal } \\
\text { without } \\
\text { disease }\end{array}$ & $\begin{array}{r}\text { fibrosis } \\
\text { associated }\end{array}$ & $\begin{array}{l}\text { Periporta } \\
\text { with } \\
\text { disease }\end{array}$ & $\begin{array}{l}\text { fibrosis } \\
\text { sociated }\end{array}$ \\
\hline \multirow[t]{2}{*}{75} & \multicolumn{2}{|c|}{$55(73.3 \%)$} & \multicolumn{2}{|c|}{$20(26.7 \%)$} \\
\hline & $\begin{array}{l}\text { Alive } \\
47(85.5 \%)\end{array}$ & $\begin{array}{l}\text { Died } \\
8(14.5 \%)\end{array}$ & $\begin{array}{l}\text { Alive } \\
13(65 \%)\end{array}$ & $\begin{array}{l}\text { Died } \\
7(35 \%)\end{array}$ \\
\hline
\end{tabular}

In this cohort $63(61 \%)$ patients had Sengestaken's tube as initial method for bleeding control, 20(19.6\%) patients out of them died. Eighty-five $(83.3 \%)$ patients underwent emergency sclerotherapy, $13(12 \%)$ of them died. Only $2(1.9 \%)$ patients underwent band ligation, with no death reported among them.

The ICU stay for the whole studied group ranged from 1 to 8 days with mean $( \pm \mathrm{SD}) 2.4$ days $( \pm 1.64)$. The ICU stay in those who died, ranged from 1 to 8 days with mean $( \pm D S) 3.4$ days $( \pm 2.1)$. There were $17(16.7 \%)$ patients with ICU stay $\geq 4$ days; $10(58.8 \%)$ out of them died. In contrast, those with ICU stay $<4$ days were $85(83.5 \%)$ patients and only $17(20 \%)$ of them died. The mean modified APACHE II score for those having ICU stay $\geq 4$ days was 17.1 , while for those having ICU stay $<4$ days was 14.7.

\section{Discussion}

Mortality risk prediction in patients admitted to ICU is commonly used in clinical research and practice ${ }^{4}$. It helps to improve accuracy when evaluating management policies, refine indications for admission to ICU and facilitate comparison of actual and predicted mortality in different series. Prediction of the outcome of patients admitted with massive variceal bleeding is difficult because of complexity of changing variables in the critical bleeder who is suffering from chronic liver disease and acute haemodynamic instability as well as other co-morbid diseases. Therefore, this complexity, suggests that a scoring system other than the simple Child-Pugh may improve the accuracy of prediction.

APACHE scoring system is widely used as an index of illness severity, for outcome prediction, research protocols, assessing ICU performance and quality of care. Despite its wide spread use little is known about its validity in patients admitted to ICU with massive acute variceal bleeding. We have done a comprehensive PubMed search and to the best of our knowledge there is no reported study comparing outcome of massive acute variceal bleeding in periportal fibrosis and cirrhosis. The present study attempted to evaluate the severity of acute variceal bleeding using locally modified APACHE II score.

In this study the mean predicted mortality was $22.8 \%$, while the actual mortality was $26.5 \%$. Therefore, our modified APACHE II score predicted mortality with $86 \%$ accuracy. The predicted mortality is lower than actual mortality because most of the patients referred from other hospitals and APACHE II is subjected to time factor bias. These findings are in keeping with the literature that delays in admission and varying referral methods may result in an inaccurate prediction of outcomes.

The mean modified APACHE II score among the deceased group was 19 while in the survival group was 13.6. This difference is statistically significant $P 0.0001$. The deceased group had significantly greater modified APACHE II score than survivals and this is obviously similar to other reports in the literature ${ }^{6-10}$. Our study demonstrated that low modified APACHE II score predicts survival while high score predicts mortality.

Patients with modified APACHE II score $\geq 15$ were $54(52 \%)$, out of them $23(42.6 \%)$ died. This means that there is increased risk of death when the score is $\geq 15$. This is very much comparable with the results of the study, which was conducted by Being Wang et a ${ }^{11}$. in patients with bleeding gastric ulcer. Also our findings are comparable to that of Sein and Gecelter ${ }^{12}$. The cutoff score of 15 has appropriate similar pattern of risk definition according to APACHE II score as was demonstrated in peritonitis ${ }^{13}$.

There were 27 patients with liver cirrhosis. 12 out of them died with actual mortality rate of $44.4 \%$. In this group of patients, the mean modified APACHE II score was 20, with mean predicted mortality $27.3 \%$. Obviously the modified APACHE II score under estimated the risk of death in this group of patients. This could be explained by relative reasonable early stability subsequently followed by decompensation of the liver. On the other hand, applying the Child-Pugh classification to the patients who died from this particular group showed class $\mathrm{C}$ in nine patients, class B in two patients and class A in only one patient. The risk of death in patients with liver cirrhosis seems to correlate better with the ChildPugh classification as a disease specific scoring system as reported previously ${ }^{14}$. In contrast there were 75 patients with periportal fibrosis, 15 patients out of them died, with actual mortality 
rate $20 \%$. They showed mean modified APACHE II score 17.7 , which is equal to mean predicted mortality $20 \%$. Whereas in the same group, patients who died have Child-Pugh class B in 8 patients $(53.3 \%)$, class C 5 patients $(33 \%)$, and 2 patients with Child class A (13.3\%).

The relationship between co-morbid diseases and death in 27 patients with liver cirrhosis in this study showed, $21(77.8 \%)$ patients have no associated disease and six $(22.2 \%)$ patients with associated diseases. In the former group nine $(42.9 \%)$ patients died, while three $(50 \%)$ patients from the latter group died. Although the figures are rather small in this study these high mortality rates obviously show that the risk of deaths is high among patients with liver cirrhosis irrespective of the absence or presence of associated diseases.

In contrast, the total number of patients with periportal fibrosis was 75 patients, 55 (73.3\%) patients of them had no associated disease, eight $(14.5 \%)$ patients from this group died. While patients with periportal fibrosis and co-morbid diseases were $20(26.7 \%)$ patients ended with seven $(35 \%)$ deaths. This observation shows that the co-morbid diseases increase the risk of death in patients with periportal fibrosis presenting with variceal bleeding.

In this study $63(61 \%)$ patients had Sengestaken's tube as first means for bleeding control, $20(19.6 \%)$ patients out of them died $(19.6 \%)$. All patients who had Sengstaken's tube had significant bleeding at presentation. Such severe bleeding is commonly associated with shock, agitation or pre-coma which precludes sclerotherapy as first step in management. The above figures demonstrate that there is correlation between massive bleeding and death. On the other hand $85(83.3 \%)$ patients underwent emergency sclerotherapy, $13(12 \%)$ out of them died. Only two $(1.9 \%)$ patients underwent rubber band ligation, with no death reported within this group. Band ligation has high cost, most of patients are farmer and too poor to pay for banding. For this reason such facilities are not always available in this centre, which deal with mass treatment with possible minimum cost.

The mean ICU stay for the whole studied group was 2.4 days. In comparison those who died had mean ICU stay 3.4 days. When we took 4 days as cutoff period we found that $17(16.6 \%)$ patients had ICU stay $\geq 4$ days with $10(58.8 \%)$ deaths, while those with ICU stay $<4$ days were $85(83.5 \%)$ patients. $17(20 \%)$ patients out of the later group died. Also the mean modified
APACHE II score for those who had ICU stay $\geq 4$ days was 17.1 , while for those who had ICU stay $<4$ days was 14.7. This shows clearly that patients with increase ICU stay $\geq 4$ days had increased mortality rate and mean modified APACHE II score, which compares well with the study conducted by Siddiqui $\mathrm{S}$, et $\mathrm{al}^{15}$.

We used locally modified APACHE II score, but looking in literature our study is similar to study conducted in Nigeria by A.R.K Adesunkanmi et al who evaluate the use of locally modified APACHE II in African children with acute generalized peritonitis ${ }^{16}$. They dropped off the blood PH and oxygen tension and they scored the absent parameter as zero in accordance with recommendation of Meakin's et al ${ }^{17}$. Because of high cost of repeated investigations in the first 24 hours most investigations were done once at admission and collection of data on admission only is economically effective, as compared to study done by HO KM et $\mathrm{al}^{18}$. They compared the admission and the 24 hours APACHE II worst scores in predicting hospital mortality and they found that substitution of 24 hours worst physiological variables with admission physiological variables maintained the over all discrimination ability of APACHE II in predicting mortality.

\section{Conclusion}

The modified APACHE II score is an effective tool in predicting outcome in patients with severe variceal bleeding. It is particularly useful in estimation risk of death in patients with portal hypertension and acute variceal bleeding secondary to periportal fibrosis. The modified APACHE II under predicts mortality by two folds in patients with variceal bleeding secondary to liver cirrhosis, which is better be scored by the Child-Pugh classification. Modified APACHEII score of $\geq 15$ points is associated with high mortality. Long ICU stay, and Child-Pugh class C at presentation increase risk of death.

\section{References}

1. Knaus WA, Zemmerman JE, Wanger DP, et al. APACHE II. Acute physiology and chronic health evaluation: A physiological based classification system. Crit Care Med $1984 ; 12: 975-7$

2. Knaus WA, Zemmerman JE, Wanger DP. APACHE II a severity of disease, classification system. Crit Care Med $1985 ; 13: 818-29$.

3. Knaus WA, Zemmerman JE, Wanger DP. Prognosis in acute organ-system failure. Ann Surg 1985; 12: 685-93

4. Barriere SL, Lowry SF. An overview of mortality risk prediction in sepsis. Crit care Med 1995; 23:376-393 
5. Dragsted L, Jorgensen $J$, Jensen $\mathrm{N}-\mathrm{H}$, et al. Interhospital comparisons of patient outcome from intensive care: importance of lead-time bias. Crit Care Med 1989; $17: 418-22$.

6. Zhonghua YI, Xue Zazhi. Predicting of in hospital mortality in patients with myocardial infarction using APACHE II system. Zhonghua Yi Xue Za Zhi (Taipei) 2001; 64:501-6

7. Koperna TI, Semmler D, Marian F. Risk stratification in emergency surgical patients: is APACHE II score a reliable marker of physiological impairment. Arch surg 2001:136; 55-59

8. Bhagwenjee S, Paruk F, Moodley $j$, et al. Intensive care unit morbidity and mortality from eclampsia: An evaluation of APACHE II score and Glasgow coma scale score. Crit Care M. $2000 ; 28: 120-4$

9. Oh JE, Hutchinson R, Short S, Bukley T, et al. Verification of acute physiology and chronic health evaluation scoring system in Hong Kong intensive care units. Crit Care Med. 1993; 21: 698-705

10. Ratanarat R, Thana Kittiwirum M, Vilai chone W, et al. Prediction of mortality by using the standard scoring systems in medical care unit in Thailand. J Med Assoc Thai. 2005; 88: 949-55

11. Being X, Wong W, King T. APACHE II score useful tool for risk assessment and aid to decision making in emergency operation for bleeding gastric ulcer. J Am Coll Surg. 1998; 187(3):287-94
12. Schein $M$, Gecelter S. APACHE II score in massive upper gastro intestinal heamorrhage from peptic ulcer, prognostic value and potential clinical application: $\mathrm{Br} \mathrm{J}$ surg $1989 ; 76: 733-736$

13. Kopernat T, Schultz F. Prognosis and treatment of peritonitis: do we need new scoring system? Arch Surg $1996 ; 13: 180-186$.

14. Chatzicostas C, Roussomoustakaki M, Notas G, et al. A comparison of child Pugh, APACHE II and APACHE III scoring systems in predicting hospital mortality of patient of liver cirrhosis . BMC Gastroenterol. 2003; 3: $7-10$.

15. Siddiqui S, Ahmed S, Manasia R. APACHE II score as predictors of length of stay and out come in our ICU. Jpa Med Assoc: $2005 ;$ 55: 253-4

16. Adesunkanmi AR, Oseni SA, Adejuyigbe O, et al. Acute generalized peritonitis in African children: assessment of severity of illness using modified APACHE II score. ANZ J Surg 2003; 73(5): 257-9.

17. Meakins JL. Solomkin S, Allo MD, et al. A proposed classification of intra-abdominal infections. Stratification of etiology and risk for future therapeutic trials. Arch Surg-1984; 119; 1372-8

18. Ho KM, Dobb GJ, Knuiman M, et al. Comparison of admission and worst 24 hours Acute physiology and chronic health evaluation II score in predicting hospital mortality. Crit Care 2006; 10:4-8. 